\title{
A Novel Data Visualization Model Based on Autoencoder Using Big Data Analysis and Distributed Processing Technology
}

\author{
Hui Feng (iD) and Guozhen Chen \\ Apparel \& Art Design College, Xi'an Polytechnic University, Xi'an, Shaanxi 710048, China \\ Correspondence should be addressed to Hui Feng; 20040725@xpu.edu.cn
}

Received 27 November 2021; Revised 24 December 2021; Accepted 27 December 2021; Published 17 January 2022

Academic Editor: Tongguang $\mathrm{Ni}$

Copyright (C) 2022 Hui Feng and Guozhen Chen. This is an open access article distributed under the Creative Commons Attribution License, which permits unrestricted use, distribution, and reproduction in any medium, provided the original work is properly cited.

\begin{abstract}
From the standpoint of visual elements, this article investigates the use of visual information technology in visual communication design. At this time, information visualization and data visualization are widely used to display visual form, which greatly facilitates people's use, provides a solid application foundation for visual communication design, and promotes its development. The image presentation of data is a common encoding process, and the reading of image content is the corresponding decoding process from the perspective of encoding and decoding. The combined efficacy of data encoding and image decoding determines the effectiveness of data visualization. It is worth noting that when it comes to "encoding and decoding," it has been established that the design mode of data visualization and visual communication is not a process of copying images but rather an external form of human thought. Then, there is the unmistakable presence of something unseen in the encoding and decoding processes. It also serves as the encoding and decoding key in the human brain. The image is as follows. From the standpoint of encoding and decoding, this article employs the data visualization self-encoder method to obtain visual data. Design pattern representation for perceptual communication can effectively support users' rapid motion analysis during the browsing process.
\end{abstract}

\section{Introduction}

The torrent of data conversion will become a trend as we move from Industry 4.0 to Industrial Internet and then to intelligent production. People are finding it more and more convenient to obtain information and data thanks to the rapid development of the Internet. Massive data quickly became a source of concern for the general public and the rate at which massive data were generated increased. This is a clear and beautiful topic in the field of visual communication [1]. Without a doubt, with the rapid advancement of technology, visual communication design will no longer be limited to a few well-known categories but will also include more related majors and make use of new science and technology to achieve a new level of separation. People's cognitive load can be reduced and a clear and intuitive visual expression can be formed using data visualization and graphical interface in an organic way [2]. Since the invention of cameras, televisions, and other tools, visual communication design has been in the process of image transformation, and interface design, as a subset of visual communication design, has also been in the process of image transformation. Vision has a significant impact on viewing data [3]. This article analyzes the application of visual information technology in visual communication design from the perspective of visual elements. At present, the display of visual form by information visualization and data visualization has been well applied, which greatly facilitates people's use, provides a certain application foundation for visual communication design, and promotes the development of visual communication design [4].

In this game, it is not a small step forward for human beings to consciously explore the rules and skills of coding from an unconscious coding, and it is also an aspect of human evolution without exaggeration. It is worth noting that when it comes to "encoding and decoding," it is affirmed that visual communication design is not a process of copying images but an external manifestation of human thought, so 
there is no doubt that there is something invisible in the human brain in the process of encoding and decoding, which is also the key to encoding and decoding. That is images $[5,6]$. From the point of view of coding and decoding, the image presentation of data is a typical coding process, and the reading of the image content is a corresponding decoding process. The effectiveness of data visualization is the unity of the effectiveness of data coding and the effectiveness of image decoding $[7,8]$. Coding shows the maximum amount of information in the shortest time and the least space, and the choice of a visualization method is very important $[9,10]$. Coding and decoding are two different things. Data information can reduce the interference of a large number of visual noises in the process of visual coding with the help of the correct visualization method, which can lead to different mining attributes of data from many angles and discovering the important information hidden in the data [11]. The real position of the current voxel in the two code tables is determined using the two-layer code table index and the corresponding offset address, and the two texture coordinates are then used to obtain the decoded value in the corresponding code table. The reconstructed data value of the voxel is obtained by adding the two decoded values and the mean value $[12,13]$. To determine the importance of data visualization in visual communication design patterns and make similar data adjacent to each other, data visualization and visual communication design patterns are coded [14].

Visual communication design under the coding and decoding theory can only take the road of diversified development, strengthen its own design, and differentiate development to participate in market competition. On this basis, it continuously interacts with, integrates, and continuously realizes innovation and improvement [15]. From the point of view of coding and decoding, the image presentation of data is a typical coding process, and the reading of the content in the image is a corresponding decoding process. The effectiveness of data visualization is the unity of the effectiveness of data coding and the effectiveness of image decoding. Simultaneously, it cultivates their desire to seek out differences, greatly improves their level of appreciation, aesthetic ability, and innovation ability, and lays the groundwork for cultivating skilled professional talents and innovative talents. To obtain the visual communication design pattern representation, a data visual self-encoder method based on the perspective of encoding and decoding is proposed, which can effectively support the rapid motion analysis of users in the browsing process. The proposed method's effectiveness is confirmed by the experimental results on the visual communication design pattern dataset.

\section{Related Work}

Literature [16] pointed out that a method of data visualization browsing uses KMeans to iteratively turn the visual communication design pattern in the data visualization set into a hierarchical tree and studies the data by specifying the starting and ending visual communication design patterns. In literature [17], using the big data analysis method and qualitative analysis of quadruple and maximum segmentation method, the visual communication design pattern is transformed into a systematic evolutionary tree, and better results of visual communication design pattern construction are obtained. Research in literature [18] showed that compared with tree organization, the visualization method of visual communication design pattern of scatter diagram based on SOM does not need to transform the relationship between nodes into a one-tomany parent-child structure. Literature [19] pointed out that the acquisition method of visual communication design pattern was introduced, and the application of data visualization in visual communication design was summarized. At the same time, the research approach of data visualization was discussed. In literature [3], through the big data analysis method, from the behavior angle of visual communication design pattern, based on the analysis method of data visualization, the characteristics of visual communication design pattern were studied, and the regularity of visual communication design pattern was excavated. Finally, the scope and demand situation of visual communication design patterns were studied. Literature [20] showed that users can finally get the desired visualization of target data by browsing. Compared with the retrieval method of example query, this method does not require users to provide query examples but also provides an important interface for users who are uncertain about the retrieval target or unfamiliar with the data field. Literature [21] pointed out that data visualization and related data are used to extract the amount of data in the process of design mode, which is used to predict the application of future visual communication design mode in data visualization and predict and analyze the visual communication design mode according to the amount of data. Literature [22] organized the analysis methods and ideas of visual communication design patterns from an application perspective using the big data analysis method, built the framework of a data visual listening system, explained the goals of data mining from the two perspectives of data and design, and explained the analysis process and mining methods of visual communication design patterns. According to literature [23], relevant methods for analyzing and studying visual communication design patterns are proposed after in-depth mining and analysis of data visualization, which aids in developing visual communication design patterns. According to literature [24], after analyzing the data of visual communication design mode, some methods for calculating the operation index of visual communication design mode are given based on data visualization. From the standpoint of encoding and decoding, this article investigates the design pattern of data visualization and visual communication. The image presentation of data is a typical coding process, and the reading of the image content is a corresponding decoding process in terms of coding and decoding. The combined effectiveness of data coding and image decoding determines the effectiveness of data visualization and visual communication design patterns. 


\section{Algorithm and Principle of Encoding and Decoding}

The coding and decoding processes are asymmetric, and the propagation process is a circular circle rather than a line, with multiple interconnected but distinct links. Visual design and information transmission are the corresponding coding and decoding processes in the field of data images. Designers imbue images with inherent and intrinsic meanings. Although the decoding of data images based on images and visuality gives the impression that "seeing is believing," it leads to the delusion that image symbols are equivalent to real-world objects. The audio signal is first passed through an analysis subband filter bank, which is the first step in coding. With a reasonable time and frequency decomposition rate, the filter bank divides the input signal into 32 equidistant frequency subbands and extracts the subband sampling signal with a factor of $1 / 32$. The extracted result from whole subband sampling is equivalent to the source signal, but there is some distortion. In a frame, there are 152 PCM audio samples, so each subband has 36 samples. The filter bank's output is calculated using the following equation:

$$
S t[i]=\sum_{k=0}^{63} \sum_{j=0}^{7} M[i][k] *(C[k+64 j] * x[k+64 j]),
$$

where $S t[i]$ is the sampling output of subband I at time $t$, $C[n]$ is the coefficient of 512-point analysis window defined in the standard, and $x[n]$ is the audio sampling input read from a 512-point sampling buffer. However, $M[i][k]=\cos [(2 * i+1) *(k-16) * \pi / 64]$ is the coefficient of the analysis matrix.

The above formula is transformed into an easy to understand convolution form to facilitate analysis:

$$
S t[i]=\sum_{n=0}^{511} x[t-n] * H i[n]
$$

where $x[t]$ is the audio sample $H i[n]=h[n] * \operatorname{Cost}[(2 * i+$ $1) *(n-16) * \pi / 64]$ at time $t$, and $h[n]=-C[n]$, when the integer part of $n / 64$ is odd. $=C[n]$, when the integer part of $n$ / 64 is even; $n$ is from 0 to 511 .

In order to adapt to this change, the training sample set is updated by adding new samples and excluding old samples. A reasonable time interval $T$ is selected. At time $t$, there is $\mathrm{XR}=\mathrm{XT} \ldots X(\mathrm{~T}-\mathrm{T})$. When new samples arrive, the training set has to be updated $\chi$ Tand reestimate $p(\vec{x} \mid X T, B G+F G)$. However, some values from old samples may belong to foreground objects, so we should use $p\left(\vec{x}{ }^{t}, B G+F G\right)$ estimation to use GMM with $m$ components. The corresponding formula is as follows:

$$
p\left(\vec{x}^{t} \mid \mathrm{XT}, \mathrm{BG}+\mathrm{FG}\right)=\sum_{m=1}^{M} \hat{\pi}_{m} N\left(\vec{x}, \vec{\mu}, \hat{\sigma}_{m}^{2} I\right)
$$

where $\vec{\mu}_{1} \ldots \vec{\mu}_{M}$ represents the average value estimated by the encoding and decoding component; $\vec{\sigma}_{1} \ldots \vec{\sigma}_{M}$ represents the variance estimated by the encoding and decoding component; the covariance matrix is assumed as the diagonal unit matrix $I$; the weight is expressed by $\hat{\pi}_{m}$, and it is nonnegative and the sum of the weights is 1 . Given a new sample at time $t$, the encoding and decoding model is updated using the following formula:

$$
\begin{aligned}
& \hat{\pi}_{m} \longleftarrow \hat{\pi}_{m}+\alpha\left(o_{m}^{(t)}-\hat{\pi}_{m}\right), \\
& \vec{\mu}_{m} \longleftarrow \vec{\mu}_{m}+o_{m}^{(t)}\left(\frac{\alpha}{\hat{\pi}_{m}}\right) \vec{\delta}_{m}, \\
& \hat{\sigma}_{m}^{2} \longleftarrow \hat{\sigma}_{m}^{2}+o_{m}^{(t)}\left(\frac{\alpha}{\hat{\pi}_{m}}\right)\left(\vec{\delta}_{m}^{T} \vec{\delta}_{m}-\hat{\sigma}_{m}^{2}\right) .
\end{aligned}
$$

Usually, the invaded objects are represented by some clusters with a small weight, so the first B largest clusters are used to approximate the background model:

$$
p\left(\vec{x}^{t} \mid X T, B G\right) \sum_{m=1}^{B} \hat{\pi} m N\left(\vec{x}, \vec{\mu}_{m}, \hat{\sigma}_{m}^{2} I\right) .
$$

If the components corresponding to each pixel are arranged in descending order of weight, you can get

$$
B=\operatorname{argmax}_{b}\left(\sum_{m=1}^{M} \hat{\pi}_{m}\left(1-c_{f}\right)\right)
$$

where CF represents a maximum scale value that belongs to the foreground object but does not interfere with the background model. For example, if a new object enters the scene and remains stationary in the scene for a period of time, the object is likely to generate an additional stable cluster. Due to the occlusion of the background, the weight $\pi_{B+1}$ of the additional cluster will continue to increase. If the object remains stationary for a long enough time, the corresponding weight will slowly exceed CF and it will be regarded as the background.

Given data $x_{1}: T, x_{i}$ is the $i$ th, $t$ is the total number of data contained, and its corresponding hidden state is $Z_{1}$ : $t$. In this article, $z_{i}$ is the projection coordinate on the two-dimensional plane. Assuming the positive and negative directions, the conditional probability distribution of $z_{t}$ about $X$ can be expressed as $P\left(z_{t} \mid x_{1: t}, x_{t: T}\right)$, where $x_{1}: T$ is the time positive direction and $X_{t}: T$ is the time reverse direction. According to VAE theory, we need to find a distribution $Q\left(z_{t} \mid x_{1: t}, x_{t: T}\right)$ to approach cc by KL divergence $P\left(z_{t} \mid x_{1: t}, x_{t: T}\right)$, and KL divergence is defined as $q$ and $\mathrm{p}$ :

$$
\begin{aligned}
D_{K L}(P, Q)= & \int Q\left(z_{t} \mid x_{1: t}, x_{t: T}\right) \log \frac{Q\left(z_{t} \mid x_{1: t}, x_{t: T}\right)}{P\left(z_{t} \mid x_{1: t}, x_{t: T}\right)} d z_{t} \\
& +D_{K L}\left(Q \| P\left(z_{t}\right)\right)-E_{z t \sim Q} \log P\left(x_{t} \mid z_{t}, x_{1: t-1}, x_{t+1: T}\right) .
\end{aligned}
$$

If the first term of equation (7) is a fixed term, minimize $\mathrm{D}$ and $(\mathrm{P}, \mathrm{q})$ can be transformed into maximization:

$D_{K L}(P, D)=-D_{K L}\left(Q \| P\left(z_{t}\right)\right)+E_{z t \sim Q} \log P\left(x_{t} \mid z_{t}, x_{1: t-1}, x_{t+1: T}\right)$. 
Equation (8) is also called the variational lower bound. Suppose that $P\left(z_{t}\right)$ obeys the distribution of $N\left(z_{t}: 0, I\right)$ and $\mathrm{Q}\left(z_{t} \mid x_{1: t}, x_{t: T}\right)$ obeys the distribution of $N\left(z_{t} ; \mu_{t}, \sigma_{t}\right)$, where $\mu_{t}=f_{\text {enc }}\left(x_{t}\right), \sigma_{t}=g_{\text {enc }}\left(x_{t}\right)$; then, $-D_{K L}\left(Q \| P\left(z_{t}\right)\right)$ in formula (4) is

$-D_{K L}\left(Q \| P\left(z_{t}\right)\right)=\frac{1}{2} \sum_{j=1}^{J}\left[1+\log \left(\sigma_{t}^{i}\right)^{2}-\left(\mu_{t}^{j}\right)^{2}-\left(\sigma_{t}^{j}\right)^{2}\right]$.

Equation (7) can be calculated by sampling from $Q\left(z_{t} \mid x_{1: t}, x_{t: T}\right), z_{t}^{l}$ is the implicit vector of sampling, and the second term can be approximately as follows:

$$
\begin{aligned}
E_{z_{r} \sim Q} & \log P\left(x_{i} \mid z_{r}, x_{t z-1}, x_{l+1 r}\right) \\
& \approx-\frac{1}{L} \sum_{j=1}^{L}\left\|x_{t}-h_{\mathrm{dec}}\left(z_{t}^{l}, x_{1: t-1}, x_{t+1: T}\right)^{2}\right\|+c .
\end{aligned}
$$

According to the above analysis, the variational lower bound of equation (7) can be expressed by equations (8) and (9). In this article, fenc and genc are coding functions, and hdec are decoding functions, which are similar to those in the BiLSTM neural network. The network structure of the decoding algorithm is shown in the figure.

In order to better adapt to the change of environment, replace equation (4) with equation (6), and the updated formula of weight can be obtained as follows:

$$
\hat{\pi}_{m} \longleftarrow \hat{\pi}+\alpha\left(o_{m}^{(t)}-\hat{\pi}_{m}\right)-\alpha c T .
$$

Among them, $c T=c / T, c$ corresponds to the number of samples supporting a Gaussian component; for example, $\alpha=1$ / $T$ can be selected, then at least $c=0.01 * T$ samples are needed to support a component, and then $c t=0.01$ can be obtained, and the specific flow corresponding to GMM is shown in Figure 1. The network structure diagram of the decoding algorithm is shown in Figure 2.

\section{Methodology}

4.1. Data Visualization and Visual Communication Design Pattern. The deeper reason for data visualization and visual communication design pattern lies in the mutual separation of "form metallurgy" and "content metallurgy." In the dimension of the visual design of data images, the content is deprived of form by strong governance, and designers have to go to great pains to find the most perfect form. In the dimension of data image content transmission, the audience has to strive to understand the content across the form, and the form realizes the separation of the content in a figurative manner. The development of data visualization and visual communication design pattern has gone through the process from static to dynamic and then to interactive presentation. The improvement of data visualization can obviously slow down the disharmony of visual communication design pattern, but it is still limited by the time period. Interaction in advance and interaction in the event are still needed; that is, analysis of the audience in advance and strengthening emotional communication in the event are needed. In order to verify the effectiveness of the coding and decoding model and algorithm, we first use the data visualization in the benchmark scene to train and test the model of our visual communication design pattern, compare the performance of network models at different depths, and compare it with the original GMM algorithm and the current advanced algorithm. Then, we further fine-tune and test the visual communication design pattern model in some new scenes. From the perspective of encoding and decoding, designers need to "design rationally"; specifically, based on data visualization, set design according to the purpose, highlight visual communication of design pattern information, match it with secondary information, and then present it aesthetically. "Reasonable" design is based on the fact that the visual communication design mode has at least the general level of image design ability. The "image garbage" and "image mediocrity" caused by "over-smelting" and "under-smelting" are not desirable. It should be noted that "reasonable" design does not require high data density image design or other high-level design, but it does not exclude the design for specific objects and specific purposes. Because there are many data visualization information and complex relationships, directly visualizing all data will lead to bloated graphics and difficulty of reading; simplifying data visualization will affect the completion of information performance, integrity, and accuracy. In the face of specific requirements, or focus on visual design, or focus on content communication and purpose, data visualization and visual communication design patterns can be finely designed to achieve specific effects. At the same time, based on the diversified needs of data visualization, visual communication design patterns can also be designed for specific data or nonspecific data. Firstly, this part analyzes the steps of data visualization and visual communication design pattern coding, which is described based on diagrams. Firstly, the input data visualization is based on a visual communication design pattern that divides the input signal into 32 equidistant frequency subbands. The sampled signal after this process is still in the time domain. Then, the sampled signal is mapped to the frequency domain by an improved discrete cosine transform. At the same time, the input PCM signal is transformed by FFT and then passed through a psychoacoustic model to judge the signal mask ratio of each subband, as shown in Figure 3.

The design pattern of data visualization and visual communication is a process of graphic simplification, which is a requirement for designers, and the information reading of data images is a complicated process, which is a requirement for audiences. Only when the requirements are clear can the problems to be solved be clearly defined and the design have an accurate direction. In the design mode of visual communication, many methods are widely used to analyze data visualization, such as interviews with users, situational investigation, auxiliary understanding of virtual objects' roles, and organizing targeted discussions among users. Referring to the training process of segnet, both models use the cross-entropy function as the loss function and use the random gradient descent algorithm to train on the Caffe framework. When training subnet-4, the 


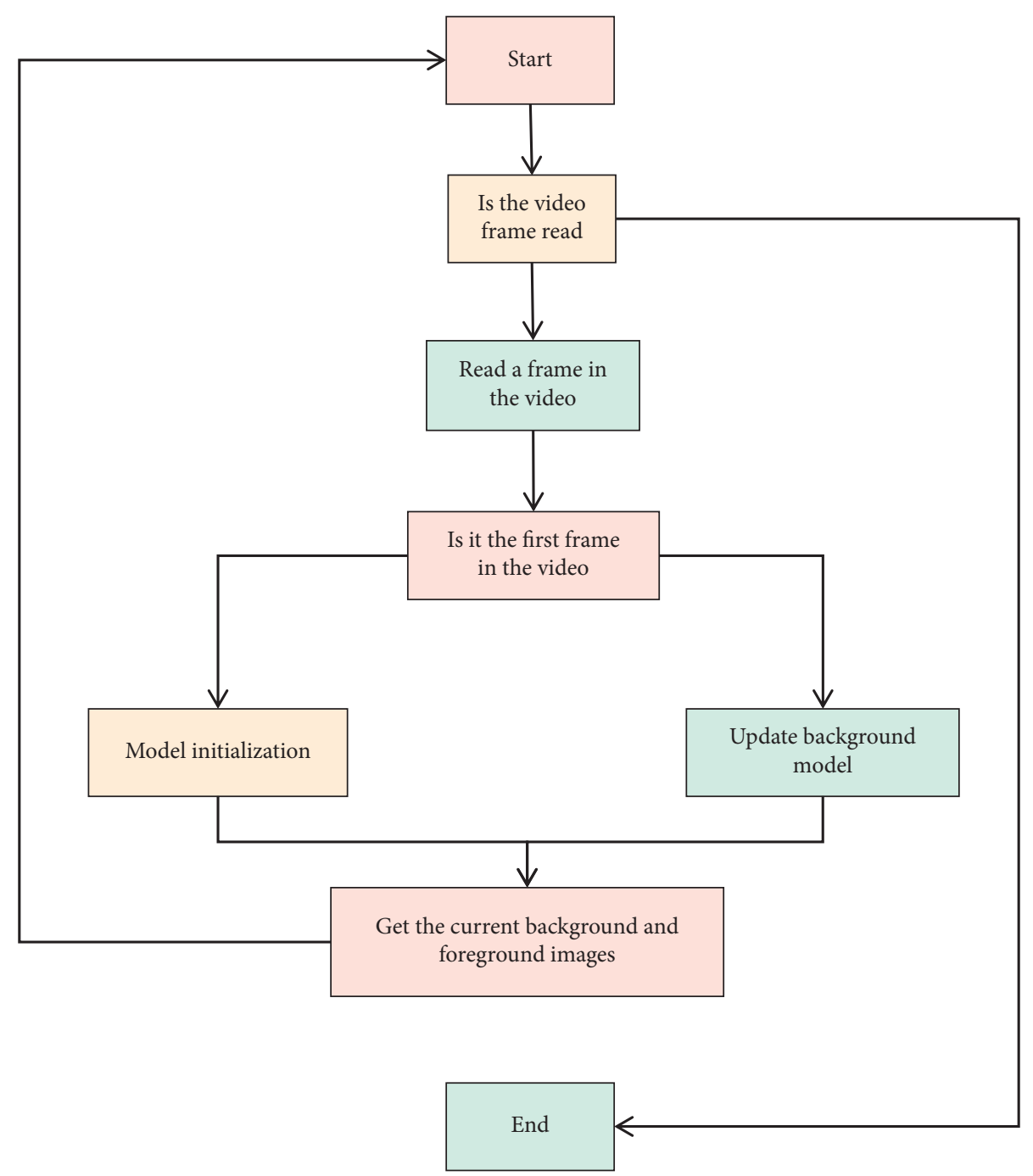

FIGURE 1: Flowchart of encoding and decoding model.

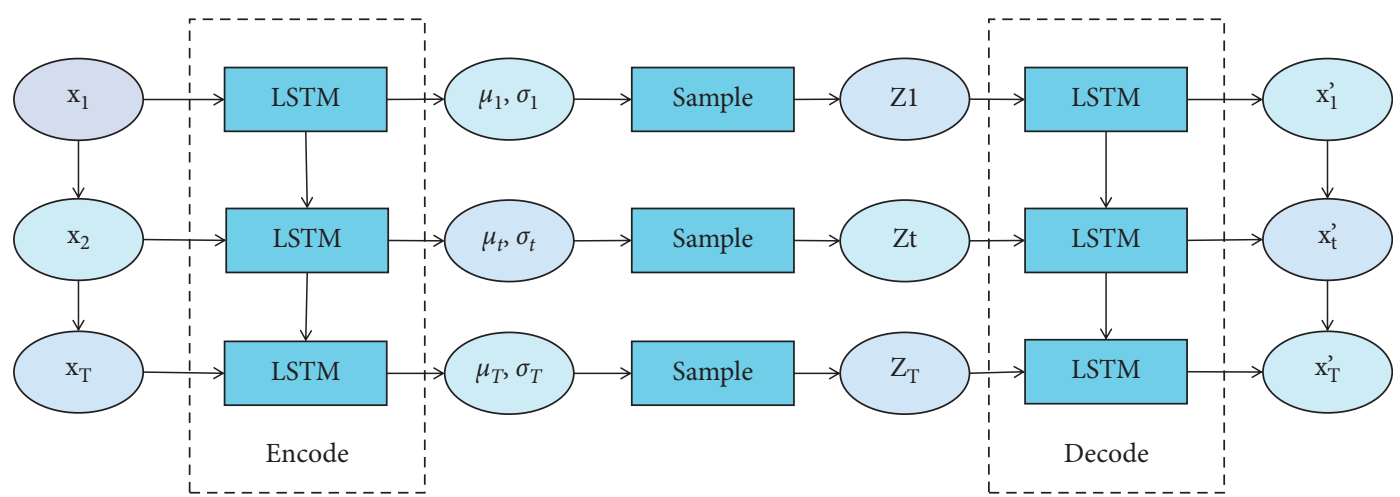

FIgURE 2: Network structure diagram of encoding and decoding algorithm.

learning rate is fixed at 0.01 and the batch size is set to 10 according to the actual hardware conditions; when training subnet-13, the learning rate is fixed to 0.001 and the batch size is set to 4 . We observed that after about 15 training cycles (epoch, which means that all training data are trained once), the two visual communication design pattern models have basically converged. In order to compare the performance of models with different depths, we further trained the two visual communication design pattern models to about 30 cycles and then tested them on the resulting visual communication design pattern model. The visual change process of loss data during the training of two visual communication design pattern models is shown in Figure 4. 


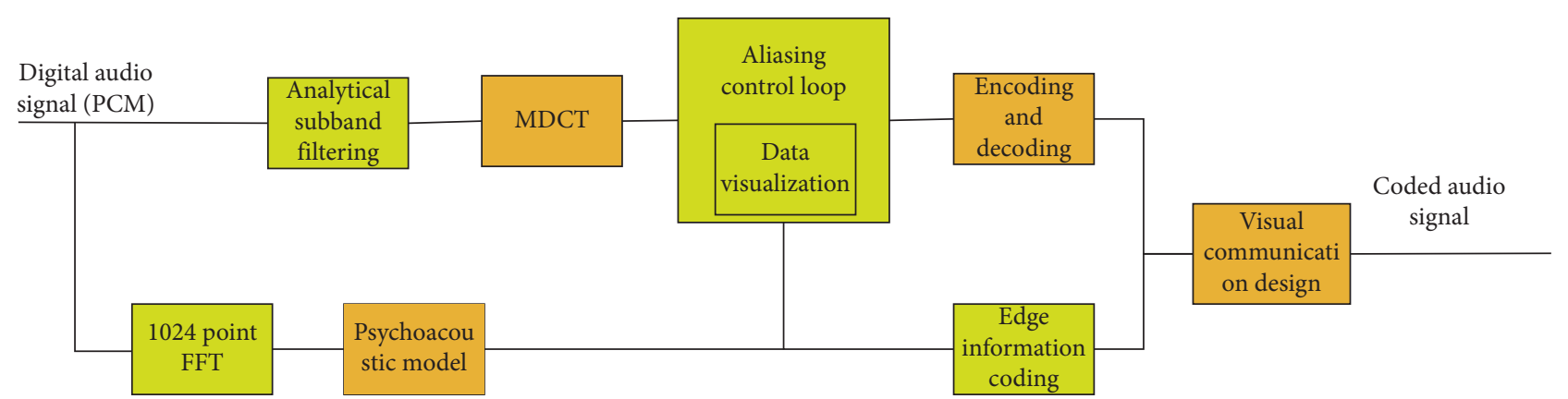

FIgURE 3: Block diagram of data visualization and visual communication design pattern coding.

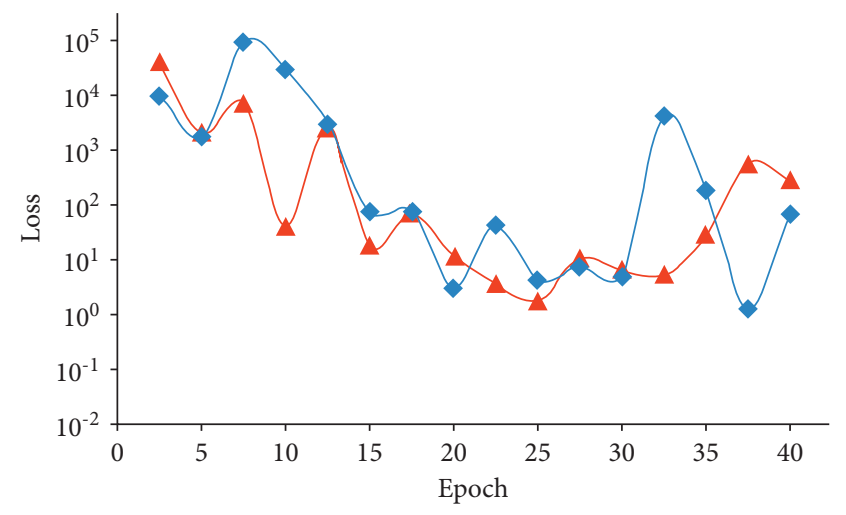

- SubNet-4

$\neg$ SubNet-13

FIGURE 4: Numerical change curve of data visualization in visual communication design mode during training.

4.2. Experimental Results of Encoding and Decoding Algorithm in Data Visualization and Visual Communication Design Pattern. The coding and decoding algorithm performs similarly to the current top algorithms, and this is achieved without further optimization of the background modeling algorithm or the detection results (for example, DeepBS uses time median filtering to further process the results after obtaining the detection results), demonstrating that the coding and decoding algorithm is very competitive and has a lot of room for improvement. The advantages of data visualization and visual communication design patterns can complement and confirm each other by commenting on them or linking to the text content library. The look-up table simultaneously inputs the value of code length into an accumulator after a codeword is decoded. The accumulator serves two purposes: first, it indicates the position of the next word to be decoded in the buffer, which is calculated by adding the lengths of previous codewords; second, once all codewords have been decoded, the buffer is notified that new codewords are available from the bitstream. The look-up table's structure is made up of data pointers and memory, with the Huffman code table to be used in decoding already stored in memory. Because the encoding and decoding algorithm includes both Huffman encoding and scale factor in the main data, it can be assumed that a single-state machine can perform both Huffman and scale factor decoding at the same time. This can simplify the design code and save the hardware area, which can be realized by sharing the master data register by Huffman decoding and scale factor decoding. "We should be moderately skeptical about the reliability of the following information, cooperate with the data collection and processing personnel to check its authenticity, and then use it as the basis for making the data visualization and visual communication design mode." Regardless of the length of the codeword, the clock cycle required for decoding each codeword is the same, and the decoding time is relatively short, which is more suitable for requiring real-time decoding. Moreover, when Hoffman's code table changes, it only needs to modify the data in the look-up table, which is more convenient in terms of universality. In the interaction between data visualization and visual communication design mode, the designer has greater control because they are the supplier of image products. "The audience has no way of verifying himself." Whether or not data visualization is the source data information: first and foremost, it is not required. The image must be self-evident but separated from the visual communication design mode results in the loss of the reference object during verification; second, data visualization implies the conception and tradeoff of the visual communication design mode, and restoring the source information is thought to be denying the designer's work and the value of the information map; third, it is a goal of data visualization; fourth, it is a goal of data visualization. The second goal is to encourage in-depth thinking and sublimation. We do not want the thinking to get stuck in the tangle of visual communication design mode after "understanding." The audience, on the other hand, is not taken for granted. The audience has the ability to read and understand basic judgments of image superiority and inferiority and the ability to "vote with their eyes"; that is, pay less attention to or ignore poorly designed data images. We conducted experiments and tests on other scenes of the CDnet dataset to further verify the generalization ability of the data visualization and visual communication design pattern model from the perspective of encoding and decoding. We only used subnet- 4 to conduct related experiments here, based on the results of previous comparisons of different depth encoding and decoding networks. The performance of subnet- 4 and subnet-13 is examined in each scene of data visualization, as shown in Figures 5 and 6.

We find that the algorithm performs well in the highway scenario but poorly in the office and pedestrian scenarios. Information transmission is realized through the encoding and decoding algorithm. There is no need to investigate 


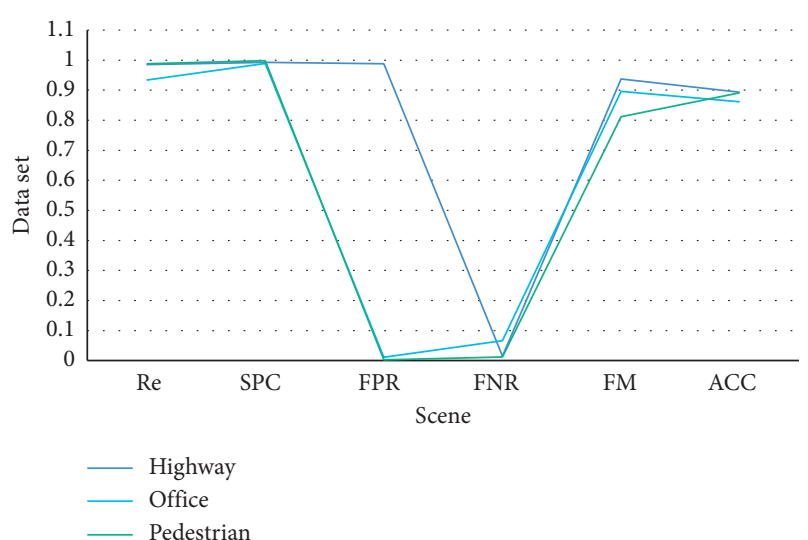

FIGURe 5: Performance of subnet-4 in various scenarios of data visualization.

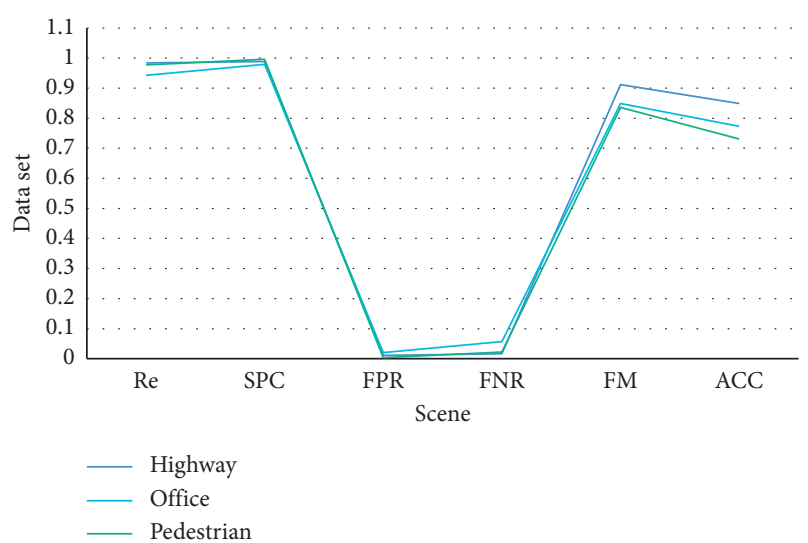

FIGURE 6: The performance of subnet-13 in various scenes in data visualization.

defects in the design process of data visualization. As long as the local visual communication design does not affect the beauty of the whole image and the audience can still understand the meaning of the image by bypassing defects, it is understandable. Of course, obvious errors are not allowed, which is related to the designer's own reasonable duty of care, including data visualization and visual communication design pattern comparison review, image ambiguity review, and aesthetic common sense review. The results show that our data visualization and visual communication design patterns are used to find the foreground object through the difference between the background image and the video frame; however, due to the shortcomings of the GMM model itself, the foreground object in the scene of data visualization and visual communication design pattern stays in the scene for a long time, which leads GMM to mistake it for the background. Considering that the design pattern of data visualization and visual communication is a scene with little background change, the subnet-4 model is used for testing, and the test results are shown in Figure 7.

First of all, we used some scenes of subnet- 4 trained in data visualization dataset under the category of data visualization and visual communication design pattern dataset badWeather, and the results are shown in Figure 8.

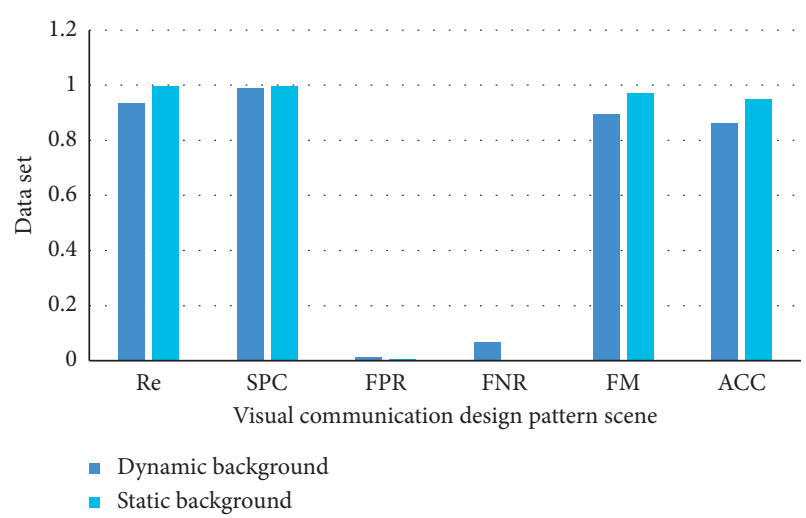

Figure 7: Performance of encoding and decoding in data visualization and visual communication design mode scenarios under different backgrounds.

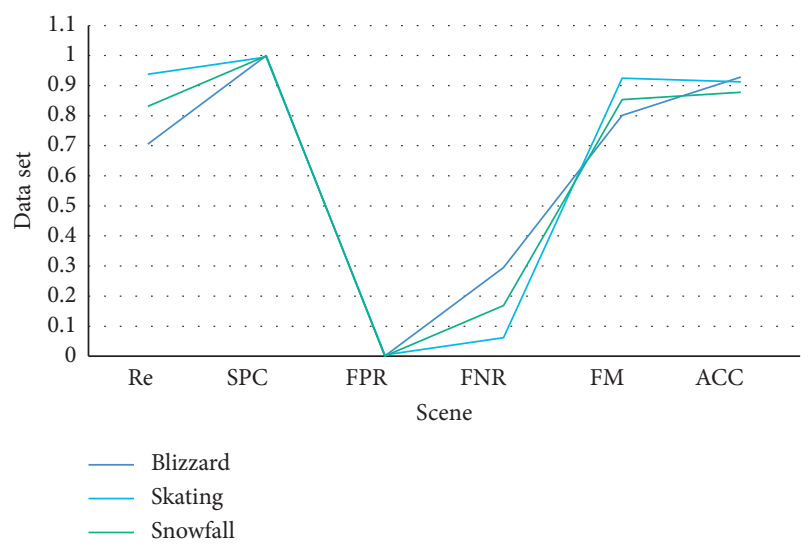

Figure 8: Performance of encoding and decoding algorithm in different scenarios of data visualization.

The results show that the encoding and decoding algorithm performs well in skating scenes but not in other scenes due to the interference of fluttering snowflakes in bad weather scenes. We may not get good results based on the contrast between the background and video frames. Using an encoding and decoding algorithm, we can fine-tune and optimize the data visualization and visual communication design pattern. In particular, we randomly selected a portion of the data from the above scenarios, taking $10 \%$ of each scenario's data, for a total of about 2000 data, and then trained and fine-tuned subnet- 4 for about 30 weeks. We tested the model in these scenarios after it converged. We also tested the average performance of the fine-tuned data visualization and visual communication design pattern model in the highway, office, and pedestrians scenes in the CDnet2014baseline category to see how fine-tuning affected the model. Figures 9 and 10 depict the test results.

It is clear from the results that the fine-tuning data visualization and visual communication design pattern model's performance in the new scene has vastly improved over the original model and that the average performance in the badWeather scene even outperforms the best existing algorithm. One of the more intriguing findings is that the fine-tuned data visualization and visual communication 


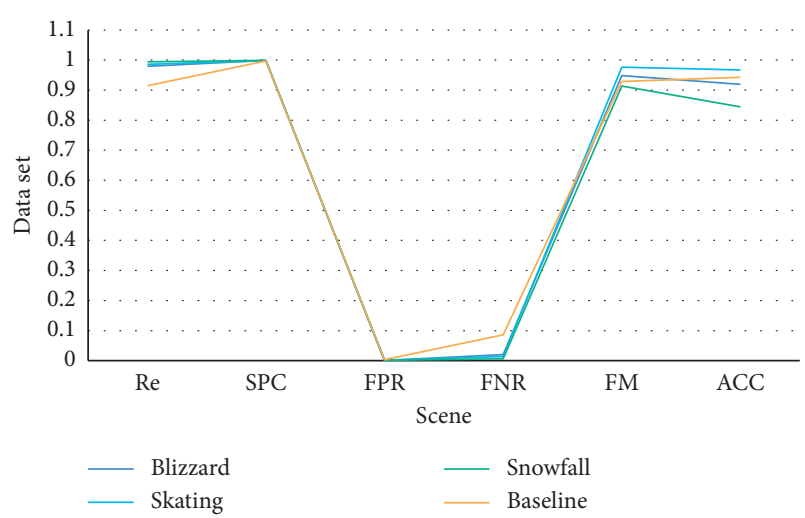

Figure 9: Performance of fine-tuned subnet-4 in various scenarios in data visualization.

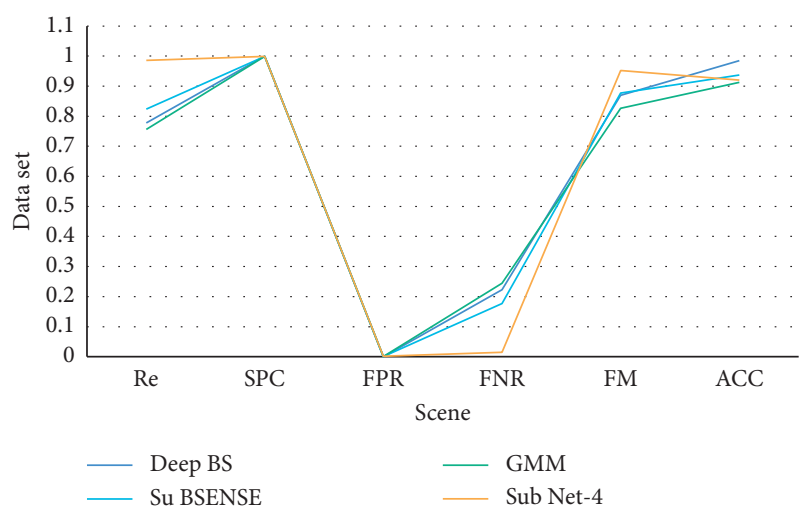

FIGURE 10: Average performance of different algorithms in some scenes of badWeather.

design pattern model's performance on the benchmark dataset has improved (the F score has increased from 0.9109 to 0.9284 ), implying that increasing the amount of data helps to improve the robustness of the data visualization and visual communication design pattern model. It is easy to see how much better the encoding and decoding algorithms are than the original GMM algorithm and how much better the antinoise ability is while effectively solving the "hole" problem.

\section{Conclusions}

Data visualization has taken off in this era of rapid advancement in computer technology and information technology, thanks to the power of science and technology. Traditional data charts have been transformed into visual communication design mode, which is of great benefit in guiding the general public to understand highly specialized data information and relieving data visualization resistance. The interactive operation mode is more user-friendly. The key to encoding and decoding is a thorough understanding of the design pattern of data visualization and visual communication, as well as the designer's improvement of drawing, the audience's adjustment, and the designer's interaction with the audience. The decoder's overall design is organized and divided into modules, with handshake signals defined. On this foundation, the decoding algorithm flow of data visualization and visual communication design pattern and the decoding system's hardware implementation scheme are thoroughly examined, and the majority of the modules are designed and simulated. The symmetry of the sine coefficient in data visualization and visual communication design pattern operation is used to improve the original algorithm, which can significantly reduce the times of addition and multiplication, based on careful analysis of the decoding algorithm of data visualization and visual communication design pattern.

\section{Data Availability}

The data used to support the findings of this study are included within the article.

\section{Conflicts of Interest}

The authors declare that they have no conflicts of interest.

\section{References}

[1] G. Pasandi, K. Mehrabi, B. Ebrahimi, S. M. Fakhraei, A. Afzali-Kusha, and M. Pedram, "Low-power data encoding/ decoding for energy-efficient static random access memory design," IET Circuits, Devices and Systems, vol. 13, no. 8, pp. 1152-1159, 2019.

[2] Z. Wang, L. Wang, S. Liu, and G. Wei, "Encoding-DecodingBased control and filtering of networked systems: insights, developments and opportunities," IEEE/CAA Journal of Automatica Sinica, vol. 5, no. 1, pp. 3-18, 2018.

[3] K. Krause, "A framework for visual communication at $\mathrm{Na}$ ture," Public Understanding of Science, vol. 26, no. 1, pp. 15-24, 2016.

[4] R. Garcia-Retamero and E. T. Cokely, "Designing visual aids that promote risk literacy: a systematic review of health research and evidence-based design heuristics," Human Factors: The Journal of the Human Factors and Ergonomics Society, vol. 59, no. 4, pp. 582-627, 2017.

[5] A. Hakone, L. Harrison, A. Ottley et al., "PROACT: iterative design of a patient-centered visualization for effective prostate cancer health risk communication," IEEE Transactions on Visualization and Computer Graphics, vol. 23, no. 1, pp. 601-610, 2017

[6] W. He, G. Xiong, K. Zhou, and J. Cai, "Reversible data hiding based on multilevel histogram modification and pixel value grouping," Journal of Visual Communication and Image Representation, vol. 40, pp. 459-469, 2016.

[7] T. Darian, "Interactive data visualization for the web: an introduction to designing with D3," Technical Communication, vol. 65, no. 3, pp. 333-334, 2018.

[8] H. Yao, C. Qin, Z. Tang, and Y. Tian, "Guided filtering based color image reversible data hiding," Journal of Visual Communication and Image Representation, vol. 43, pp. 152-163, 2017.

[9] J. D. Camm, M. J. Fry, and J. Shaffer, "A practitioner's guide to best practices in data visualization," Interfaces, vol. 47, no. 6 , pp. 473-488, 2017.

[10] H. Niu and S. Dong, "Improving boundary level calculation in quantized iterative learning control with encoding and decoding mechanism," IEEE Access, vol. 7, no. 99, pp. 1-3, 2019. 
[11] Z. Huang, Y. Liu, C. Zhan, C. Lin, W. Cia, and W. Chen, "A novel group recommendation model with two-stage deep learning," IEEE Transactions on Systems, Man, and Cybernetics: Systems, vol. 2021, Article ID 3131349, 2021.

[12] J. Wang, X. Li, and B. Ou, "Improved PVO-based reversible data hiding: a new implementation based on multiple histograms modification," Journal of Visual Communication \& Image Representation, vol. 38, pp. 328-339, 2016.

[13] S. Johnson, F. Samsel, G. Abram et al., "Artifact-based rendering: harnessing natural and traditional visual media for more expressive and engaging 3D visualizations," IEEE Transactions on Visualization and Computer Graphics, vol. 26, no. 1, pp. 492-502, 2019.

[14] A. Yamori and K. Sakai, "Video encoding method and apparatus, and video decoding apparatus," Studies in English Language and Literature, vol. 37, no. 12, pp. 159-166, 2017.

[15] T. Moriya, Y. Kamamoto, and N. Harada, "Encoding apparatus, decoding apparatus, and method and program for the same," Journal of the Acoustical Society of America, vol. 96, no. 2, pp. 1222-1224, 2017.

[16] M. Moerel, F. D. Martino, V. G. Kemper et al., "Sensitivity and specificity considerations for fMRI encoding, decoding, and mapping of auditory cortex at ultra-high field," NeuroImage, vol. 164, pp. 18-31, 2017.

[17] S. Deneve and M. Chalk, "Efficiency turns the table on neural encoding, decoding and noise," Current Opinion in Neurobiology, vol. 37, pp. 141-148, 2016.

[18] W. Hauser, "Decoding the social world: data science and the unintended consequences of communication," Information, Communication \& Society, vol. 21, no. 11, pp. 1866-1867, 2018.

[19] Z. Tian, "Dynamic visual communication image framing of graphic design in virtual reality environment," IEEE Access, vol. 8, no. 99, pp. 1-2, 2020.

[20] C. Zhang, B. Ou, H. Tian, and Z. Qin, "Reversible data hiding in JPEG bitstream using optimal VLC mapping," Journal of Visual Communication and Image Representation, vol. 71, Article ID 102821, 2020.

[21] M. M. Ali, H. Wahid, and N. M. Subha, "Data visualization for human capital and halal training in halal industry using tableau desktop," Communications in Computer and Information Science: Modeling, Design and Simulation of Systems, Springer, vol. 752, Germany, 2017.

[22] S. Nersesian and S. Bourgaize, "Illustrating your research: design basics for junior clinicians and scientists," BMJ, vol. 6 , pp. 370-372, 2020.

[23] K. B. Schloss, Z. Leggon, and L. Lessard, "Semantic discriminability for visual communication," IEEE Transactions on Visualization and Computer Graphics, vol. 99, pp. 1-8, 2020.

[24] M. Landgren, S. S. Jakobsen, B. Wohlenberg, and L. B. Jensen, "Informing sustainable building design," Archnet-IJAR: International Journal of Architectural Research, vol. 13, no. 1, pp. 194-203, 2019. 